Voix et Images

voixetimages

\title{
Greimas et Thériault
}

\section{Robert Major}

Volume 23, numéro 1 (67), automne 1997

Madeleine Ouellette-Michalska

URI : https://id.erudit.org/iderudit/201351ar

DOI : https://doi.org/10.7202/201351ar

Aller au sommaire du numéro

Éditeur(s)

Université du Québec à Montréal

ISSN

0318-9201 (imprimé)

1705-933X (numérique)

Découvrir la revue

Citer cet article

Major, R. (1997). Greimas et Thériault. Voix et Images, 23(1), 169-173.

https://doi.org/10.7202/201351ar

Ce document est protégé par la loi sur le droit d'auteur. L'utilisation des services d'Érudit (y compris la reproduction) est assujettie à sa politique d'utilisation que vous pouvez consulter en ligne.

https://apropos.erudit.org/fr/usagers/politique-dutilisation/
Cet article est diffusé et préservé par Érudit.

Érudit est un consortium interuniversitaire sans but lucratif composé de l’Université de Montréal, l'Université Laval et l'Université du Québec à Montréal. Il a pour mission la promotion et la valorisation de la recherche. https://www.erudit.org/fr/ 


\title{
Essai
}

\section{Greimas et Thériault}

\author{
Robert Major, Université d'Ottawa
}

L'œuvre d'Yves Thériault, après avoir beaucoup retenu l'attention de la critique, semble être entrée dans une forme de purgatoire depuis la mort de l'auteur en 1983. Pendant les décennies soixante et soixante-dix, les thèses et les études substantielles se multipliaient, autant au Québec qu'au Canada anglais. Cette œuvre multiple, foisonnante, fort originale et volontiers iconoclaste, avait pris d'assaut l'institution littéraire dans le courant des années cinquante, faisant éclater les cadres et les références traditionnelles. Yves Thériault semblait prendre un malin plaisir à se renouveler avec chaque titre, passant du terroir à la synagogue, du village québécois à la toundra, de la forêt boréale aux terres arides d'une Espagne mythique, du livre pour adolescents aux contes érotiques, du roman d'aventures au récit introspectif, visant tous les publics et les déroutant tous, et poussant même l'outrecuidance jusqu'à publier — dans un pays d'écriture étique et de silence accédant difficilement à la parole quatre livres la même année!

Thériault, en effet, avait plaisir à se présenter comme une force de la nature, autodidacte et conteur spontané, puisant son inspiration dans les sèves telluriques de la Nature primi- tive ou dans les tréfonds aussi sauvages de son imaginaire libertaire; ne sachant que faire, d'ailleurs, du savant ergotage de ses confrères écrivains et s'en moquant allègrement; prompt aussi à colleter ceux qu'il estimait être des intellectuels désincarnés, coupés de la vie, celle-là même que, évidemment, son ouvre à lui véhiculait sans subterfuges.

À ce titre, avec cette espèce de méfiance de l'intellectuel et de toute abstraction, sa soif éperdue de réussite matérielle et sa ferme volonté de réaffirmer ses racines primitives ou populaires, Yves Thériault est peutêtre le plus américain de nos écrivains. Entendons "américain " dans un des sens véhiculés par la tradition étatsunienne: l'opposition viscérale à l'intellectualisme "européen". Par ailleurs, cette figure de l'écrivain est en bonne partie représentation, image, construction: l'auteur a voulu faire figure, jouer un personnage. C'est le rôle qu'il a décidé d'incarner. Il ne faut pas oublier qu'Yves Thériault a commencé sa carrière comme écrivain radiophonique et annonceur polyvalent, habile à jouer tous les rôles, attiré par l'écriture dramatique et le monde du spectacle. Son ouvre est plus complexe et plus savante qu'il ne voulait le laisser entendre ou 
qu'il n'osait admettre; elle paie en retour les excellents critiques qui ont bien voulu l'aborder (je pense en particulier aux Brochu, Mailhot, Bérubé). Son éclipse relative n'est donc sans doute que cela en effet: une occultation passagère et partielle. Peut-être, d'ailleurs, la publication récente d'un livre fort savant sur le plus connu de ses romans signalet-elle le début du retour en force de l'œuvre.

\section{**}

J'aime croire qu'Yves Thériault aurait accueilli avec le plus vif plaisir le livre de Paul Perron, Semiotics and the Modern Quebec Novel: A Greimassian Analysis of Thériault's Agaguk ${ }^{1}$. Et non seulement parce qu'il maîtrisait la langue anglaise, au point même d'hésiter, en début de carrière, sur la langue de ses œuvres à venir. Le plaisir aurait été ailleurs, ambivalent et paradoxal. Tout en protestant pour la forme contre ces universitaires qui s'évertuent à tout compliquer et à lire des significations cachées dans une prose qu'il disait transparente, il aurait été flatté d'un tel déploiement. Il serait difficile, d'ailleurs, de trouver contraste plus saisissant que celui-ci, entre la simplicité professée (et factice) de l'écrivain et la démarche complexe et plutôt aride d'un littéraire soucieux d'élaborer rigoureusement une armature théorique et méthodologique cohérente pour l'analyse du texte romanesque.

Cette étude, en effet, se déploie sur Agaguk, roman que Paul Perron avait déjà étudié dans un article publié dans Voix et Images ${ }^{2}$. Mais il ne serait pas exagéré de dire que l'intention de l'auteur se situe ailleurs. Son objectif essentiel est double: d'une part, présenter à un public anglophone la théorie sémiotique de l'école de Greimas; d'autre part, essayer de démontrer la valeur heuristique de cette théorie en élaborant une méthode détaillée d'analyse ou un protocole de lecture d'un texte de la dimension d'un roman.

Le premier de ces objectifs semble exclure les lecteurs francophones et sans doute importe-t-il de se poser la question de la pertinence de cette étude (ou du moins de sa recension ici) pour la grande majorité des lecteurs de Voix et Images. Effectivement, le destinataire de Paul Perron est anglophone et non québécois. Or, indépendamment de la valeur propre de l'étude, sur laquelle je reviendrai, il y a, il me semble, un intérêt considérable à se donner le mal de lire, dans une autre langue que la nôtre, des études sur la littérature québécoise. Même quand l'auteur est francophone, comme c'est le cas ici, la médiation ou la réfraction de l'anglais (en l'occurrence) confère au texte, et surtout à son objet d'analyse, une étrangeté et donc une nouveauté absolue. C'est comme voir une personne intimement connue dans une situation radicalement imprévisible: elle nous est soudainement révélée dans une autre identité, dans un "être" qu'on ne pouvait guère soupçonner. Peut-être faudrait-il se référer aux théories sartriennes du regard: le regard de l'autre figeant l'en-soi, le chosifiant, le faisant autre; ou encore, et sans doute plus opportunément, en appeler aux théories brechtiennes de la distanciation, celle-ci étant indispensable à la réflexion critique. En 
somme, cette autre langue permet une distance, nous sort du ronron des discours connus, ceux de la petite famille québécoise. Et quand, de plus, le texte est difficile, complexe, sollicitant constamment l'attention, comment nier qu'il permet de redécouvrir (à moins qu'il ne force à comprendre). En somme, ceux qui ne sont pas de la confrérie de Greimas auraient donc probablement intérêt à lire cette étude: ils comprendront peut-être certaines choses pour la première fois!

Paul Perron poursuit donc essentiellement un double objectif théorique. Mais du fait même il présente une nouvelle lecture d'Agaguk, dont la traduction canadienne-anglaise remonte à 1963 . Le roman est inséré dans une tradition littéraire, tradition dont les moments importants sont marqués par le roman historique (Les anciens Canadiens), le roman agraire (Maria Chapdelaine), le roman urbain (Bonbeur d'occasion) et finalement le roman des espaces sauvages (the wilderness novel) dont l'exemple est Agaguk. Cet échantillonnage peut sembler banal, mais en réalité Paul Perron, dans un texte dense et de nombreuses notes substantielles, présente une lecture brève mais stimulante de ces œuvres archiconnues. En effet, cette introduction historique cherche à dégager, d'une façon schématique et dans une perspective sociosémiotique, les options qui s'offrent aux romanciers dont les œuvres, comme tous les autres artefacts culturels, doivent s'insérer dans un réseau complexe de textes et de séries culturelles. C'est la présentation sérielle qui est stimulante, et non seulement pour le public anglophone visé. Fin de la série, Agaguk est un icône de la Révolution tranquille, car il provoque une véritable révolution dans l'identification du sujet, sujet érotisé, doté d'un corps qui est la médiation obligée de son rapport au monde, et dont les rapports au groupe sont essentiellement conflictuels. La construction du sujet, sujets individuels et sujet-couple, se fait dans un espace imaginaire, absolu, quasi mythique, mais qui inscrit en filigrane l'espace québécois.

On peut donc presque lire l'introduction et la conclusion de cette étude isolément et tirer de ces relations synthétiques un profit certain. Mais l'essentiel est ailleurs. Dans la présentation synthétique de la théorie de Greimas; dans la méthode d'analyse proposée.

Je n'ai guère la compétence voulue pour juger le premier aspect. Paul Perron, d'ailleurs, n'en est pas à ses premières armes en sémiotique greimassienne, ayant traduit, commenté, expliqué cette théorie à de multiples occasions. Il en est incontestablement un spécialiste. Spécialiste peu jargonneur, toutefois, soucieux de faire comprendre et de véhiculer l'essentiel. Le deuxième chapitre me semble donc un modèle de présentation synthétique. Les fondements anthropologiques de la sémiotique, l'historique de la réflexion sur le signe, les contributions particulières de Saussure et de Hjelmslev, la théorie de Greimas et de ce qu'elle doit surtout à Propp et à Lévi-Strauss, la question spécifique d'une sémiotique littéraire et de ses conditions d'existence, les notions d'"univers sémantique", de "microunivers sémantique", d' univers du discours", d' "axiologie", d'u idéologie" sont présentés de façon succincte et cohérente. 
Les chapitres suivants ne délaissent pas la réflexion théorique ni le souci d'exposition abstraite. L'objectif étant d'élaborer une méthode d'analyse et de la mettre à l'épreuve, l'auteur est tenu, à tout moment, d'examiner ses présupposés théoriques, d'évaluer leur pertinence, de proposer une terminologie adaptée, d'effectuer des choix. Le roman est un texte long et sa simple masse linguistique pose des problèmes considérables à une théorie très complexe, plus adaptée à la micro-analyse et à une démarche hypothético-déductive. En un mot, si Greimas a consacré tout un livre, fort dense, à un bref conte de Maupassant, comment doit-on traiter un long roman?

Avec beaucoup de souplesse et de discernement, et un sens développé du relativisme de notre démarche, semble nous dire paul Perron. Le roman est une totalité signifiante qui se manifeste dans un système de signes réciproquement motivés. Il importe alors de peaufiner les techniques qui permettront de dépister les signes, de les examiner dans ce qui les constitue comme signes, de reconnaître leurs relations et leurs lois combinatoires. La question de la segmentation d'un texte aussi long est un problème particulièrement difficile. De plus, ne pouvant tout faire, Perron s'attache particulièrement au réseau des qualifications et à la grammaire narrative, et consacre un excellent chapitre à la question des valeurs, celles-ci étant centrales à une sémiotique des passions et de la cognition, et indispensables à la compréhension de l'axe syntaxique (sujet $\rightarrow$ objet). Par ailleurs, l'importance de la sexualité dans ce roman le conduit à modifier considé- rablement sa méthodologie car le corps, dans Agaguk, lui apparaît rapidement comme la médiation essentielle entre le sujet et l'objet de valeur, à la fois partie du microunivers sémantique et la perspective à travers laquelle l'univers est saisi.

\section{$* *$}

Au terme d'une telle lecture, sans doute est-on justifié de poser la question de tout Anglais pragmatique. "The proof is in the pudding": voilà une belle recette, mais qu'est-ce que cela donne dans l'assiette? Les conclusions de Paul Perron sont remarquablement sobres, à mille lieues du triomphalisme que d'autres de la chapelle ont pu afficher, à une autre époque. On pourrait les résumer ainsi: une lecture sémiotique d'un roman est forcément réductrice; une telle lecture produit un savoir essentiellement abstrait; cette méthode de lecture est complexe et peut même paraître inutilement embarrassée; par ailleurs, le modèle sémiotique est d'un intérêt théorique certain, car il décrit les éléments et les procès qui construisent la signification; ce modèle accroît indéniablement la lisibilité d'un texte, car il explique ce qui a pu être d'abord en partie compris sans l'aide de la sémiotique; la méthode sémiotique fait donc le lien entre l'épistémologie et la connaissance textuelle; la démarche sémiotique est éminemment compatible avec la variété innombrable des lectures critiques qu'on peut faire d'un texte littéraire: la sémiotique n'est pas l'essence du texte mais une des perspectives sur l'œuvre; par ailleurs, s'attachant à décrire l'objet signifiant, la sémiotique 
peut sans doute prétendre à un statut d'antériorité logique.

Ainsi, même si cette étude n'aboutit pas forcément à une nouvelle lecture critique d'Agaguk, elle explique systématiquement et rigoureusement le pourquoi des significations qui ont pu être dégagées par les lecteurs perspicaces du roman. Pour cela, pour sa valeur heuristique de jonction féconde entre théorie et texte, ce livre devrait attirer l'attention des lecteurs francophones.
1. Paul Perron, Semiotics and the Modern Quebec Novel: A Greimassian Analysis of Theriault's Agaguk, Toronto, University of Toronto Press, coll. *Toronto Studies in Semiotics *, 1996, 170 p.

2. Paul Perron, "Théorie actantielle et processus idéologique: Agaguk d'Yves Thériault ", Voix et Images, vol. IV, $\mathrm{n}^{\circ} 2$, décembre 1978 , p. 272-299. Curieusement, la bibliographie du livre ne fait pas état de cet article où avait d'abord été mise à l'essai la théorie de Greimas. Toujours dans la bibliographie, signalons qu'il faut lire Duquette, Jean-Pierre (et non Duquet). 